\title{
Ahiqar tra leggenda e rielaborazione letteraria Una tradizione e i suoi riflessi
}

\author{
Giancarlo Toloni * \\ Università Cattolica del Sacro Cuore, Brescia
}

\begin{abstract}
Ahiqar Between Legend and Literary Re-elaboration. A Tradition and Its Reflections. - The legend of Ahiqar, Sennacherib's powerful Prime Minister, is told in the homonymous History and Proverbs, whose the oldest witness is the Elephantine version $\left(5^{\text {th }}\right.$ century BC). The figure of the Assyrian wise came later in the book of Tobit as an important partner of Tobit; so, to give prestige to his book, the autor of Tobit has exploited the fame of the History of Ahiqar, and historicized its protagonist. This legend of court was also known to the Jewish communities of the Egyptian Diaspora-including that of Elephantine: in the Hellenistic period it was adapted to Jewish tradition, becoming a wisdom novel. Ahiqar also appeares in some writings in demotic: in two fragments of papyrus of the first century $\mathrm{AD}$, and in the Instructions of Anchsheshonqy, but especially in an inscription found at Uruk-Warka in a Babylonian tablet (165 BC) which contains a list of ummānu, 'scholars (of the court)'. Among them is Ahiqar, designated with an Aramaic name, Ahu'aqari. This is, therefore, an apocryphal identification: the ummānu Ahiqar is placed under Esarhaddon and in Assyria, by a spurious historicization, very similar to the one that made the author of Tobit.
\end{abstract}

KEYwORDS: Ahiqar; Elephantine; Tobit; Aramaic; Historicization; Ummānū.

AJICAR, ENTRE LEYENDA Y REELABORACIÓN LITERARIA: UNA TRADICIÓN Y SUS REFLEJOS.La leyenda de Ajicar, poderoso primer ministro de Senaquerib, aparece contada en la Historia homónima y Proverbios. Su testimonio más antiguo es la versión de Elefantina (siglo V a.C.). La figura del sabio asirio se introdujo más tarde en el libro de Tobit como un importante compañero de Tobit, de manera que para dar prestigio a su libro, el autor de Tobit explotó la fama de la Historia de Ajicar e historizó a su protagonista. Esta leyenda cortesana era también conocida entre las comunidades de la diáspora egipcia, incluida la de Elefantina; en el periodo helenístico fue adaptada a la tradición judía como novela sapiencial. Ajicar también aparece en algunos escritos en lengua demótica: en dos fragmentos de papiro del siglo III d.C. y en las Instrucciones de Anchsheshonqy, pero especialmente en una inscripción encontrada en Uruk-Warka en una tablilla babilónica (165 a.C.) que contiene una lista de ummānū, 'sabios (de la corte)'. Entre ellos está Ajicar con un nombre arameo Ahu'aqari. Es, por tanto, una identificación apócrifa: se sitúa al sabio Ajicar bajo el poder de Asarhaddón y en

\footnotetext{
*giancarlo.toloni@fastwebnet.it
} 
Asiria, por una espúrea historización, muy semejante a la que hizo el autor del libro de Tobit.

PALABRAS Clave: Ajicar; versión de Elefantina; Tobit; historización; arameo; novela sapiencial; ummānū.

La storia di Ahiqar, ${ }^{1}$ potente primo ministro di Sennacherib in epoca assiro-babilonese, è trasmessa principalmente dal libro omonimo. Nel suo insieme esso si presenta come un'opera composita. La complessità della sua fisionomia risulta già dalla titolazione tradizionale Storia e Massime/Proverbi di Ahiqar, che dà ragione delle due sezioni di cui esso consta, diverse per funzioni e per genere letterario, pur trattandosi, nei lineamenti generali, di uno scritto sostanzialmente unitario, di carattere sapienziale. Notoriamente considerato come «il libro più antico della letteratura internazionale», ${ }^{2}$ esso è attestato principalmente dalla versione di Elefantina e dalla rielaborazione delle versioni successive. Tuttavia non è questo l'unico filone della tradizione, in cui è conservata memoria della figura del saggio assiro, dato che anche in alcuni scritti in demotico e in una tavoletta di Uruk-Warka affiorano indirettamente tracce interessanti di questo personaggio singolare, emblematico, per certi versi, dell'importanza assunta dal potere politico assiro nello scenario politico del tempo. ${ }^{3}$ Lo stesso libro biblico di Tobia ne documenta l'autorevolezza, dato che l'agiografo lo inserisce direttamente nella trama del suo racconto, facendone un importante interlocutore del protagonista, Tobi, ai fini di aumentare il prestigio del messaggio del libro.

Anche di queste testimonianze collaterali si dovrà quindi tener conto nello studio della tradizione di Ahiqar e del suo protagonista, per delinearne correttamente la linea evolutiva, e individuare i tratti novellistici e romanzeschi che si sono via via aggiunti al nucleo originario

\footnotetext{
${ }^{1}$ Il nome del saggio assiro è trascritto qui per comodità senza segni diacritici, cioè Ahiqar anziché 'Ahîqqār. Questo contributo partecipa al Progetto di ricerca italiano «Il romanzo e i proverbi del saggio Ahiqar d'Assiria: origini, versioni primarie e secondarie, mutazioni e diffusione di un'opera letteraria dalla Mesopotamia antica in Oriente e Occidente» - Coordinatore Scientifico: Prof. Frederick M. Fales; Responsabili di Unità: Proff. Riccardo Contini e Fabrizio A. Pennacchietti.

${ }^{2}$ E. Meyer, Der Papyrusfund von Elephantine (Leipzig, 1912) p. 128.

${ }^{3}$ Una presentazione autorevole è offerta dalla monografia di F. M. FALEs, L'impero assiro. Storia e amministrazione (secc. IX-VII) (Roma - Bari, 2001).
} 
del racconto, aprendo la via a innumerevoli riletture che ne han reso sempre più complessa la ricostruzione critica.

\section{LA LEGGENDA E LE SUE ATTESTAZIONI}

La storia narrata nel libro di Ahiqar ha i connotati dall'esemplarità: essa adombra la diversa sorte che spetta all'uomo giusto e pio, che passa attraverso varie vicissitudini dolorose e drammatiche, ma riesce ad uscirne grazie alla sua fede incrollabile nella giustizia divina, a differenza della mala sorte, che alla fine travolge il malvagio, sebbene a questi da principio sembrino assicurate l'impunità e il successo. Questo «contenuto favoloso», insieme allo «stile piano e accattivante», ${ }^{4}$ sono all'origine della fortuna che sempre arrise al libro nel Vicino Oriente antico, forse proprio perché esso si pone a metà fra la favola e il romanzo edificante di stampo sapienziale. La Storia di Ahiqar costituisce la sezione che più richiama il confronto con Tobia, essendo un racconto condotto in forma autobiografica che inquadra in un'unica cornice due raccolte di massime e brevi apologhi di carattere gnomico, secondo un cliché narrativo molto diffuso nel Vicino Oriente antico. ${ }^{5}$ Il tema è bipartito: disgrazia e riabilitazione di un cortigiano; ingratitudine di un nipote. Entrambi i motivi stanno anche alla base del racconto in Tobia, con alcune trasposizioni degli elementi narrativi, come si vedrà.

Il protagonista del racconto è Ahiqar, primo ministro del re assiro Sennacherib, rinomato per la sua saggezza. Su consiglio della divinità, adotta il nipote Nadin (o Nadan) e provvede alla sua educazione, perché un giorno possa subentrargli a corte, non avendo egli figli. Effettivamente Nadin diverrà ministro del re dopo di lui. Ma a questo punto la narrazione

${ }^{4}$ F. A. Pennacchietti, «Storia e massime di Achicar (Introduzione, traduzione e commento)», in P. SACCHI (ed.), Apocrifi dell'Antico Testamento (Torino, 1981; rist. 1989) vol. I, pp. 53-95: 57 (traduzione e annotazioni riprodotte, con modifiche, in R. Contini - C. GrottanelLi [eds.], Il saggio Ahiqar. Fortuna e trasformazioni di uno scritto sapienziale. Il testo più antico e le sue versioni [Brescia, 2005] pp. 196-225).

${ }^{5}$ J. C. Greenfield, «The Background and Parallel to a Proverb of Ahiqar», in A. Caquot - M. Philonenko (eds.), Hommages à André Dupont-Sommer (Paris, 1971) pp. 49-59: 50-51 (= S. M. Paul - M. E. Stone - A. Pinnick [eds.], 'Al Kanfei Yonah Collected Studies of Jonas Greenfield on Semitic Philology [Leiden - Jerusalem, 2001] vol. I, pp. 93-103). 
si interrompe bruscamente, lasciando il posto a una prima sezione di proverbi, scanditi nell' esordio dalla medesima formula d'apostrofe «figlio mio», e finalizzati a suggerire le regole per giungere al successo sociale.

La vicenda riprende nuovamente con il venir meno delle aspettative che Ahiqar aveva riposto in Nadin, come attestano anche due frammenti papiracei in demotico: il nipote, ingrato, temendo di essere diseredato dal padre adottivo, lo calunnia presso Sennacherib, che ne decreta la condanna a morte. Ahiqar tuttavia riesce a sottrarsi alla pena capitale grazie all'aiuto di un collega designato come suo carnefice- cui in passato egli pure aveva salvato la vita, e si nasconde in attesa di potersi riabilitare agli occhi del sovrano. Nel frattempo il faraone d'Egitto approfitta della morte presunta del potente primo ministro assiro per imporre un grave tributo a Sennacherib che, incapace di fronteggiare la situazione, rimpiange di non potersi più avvalere, come in passato, del saggio consiglio di Ahiqar, ma, quando apprende che questi è sfuggito alla morte, lo fa richiamare a corte e lo reintroduce nel suo ruolo.

Quel che segue non ha riscontro nel libro di Tobia; se ne coglie un'eco invece nell'opera sapienziale in demotico nota col titolo di Istruzioni di Anchsheshonqi. Si tratta del viaggio di Ahiqar alla corte del faraone per risolvere l'incidente diplomatico creatosi con l'Assiria: grazie alla sua saggezza, egli è in grado di esaudire le varie richieste e i quesiti con cui il faraone lo mette alla prova, e ottiene la revoca del tributo. Al suo ritorno in Assiria si dedica alla punizione di Nadin, col consenso di Sennacherib, e rivolge così al nipote una seconda raccolta di massime: brevissimi apologhi, diversi dai precedenti per la loro natura di requisitoria, e aventi come soggetto per lo più animali od oggetti.

\section{IL PROBLEMA DELLE FONTI}

La più antica versione a noi pervenuta di Ahiqar è quella del papiro di Elefantina (V sec. a.C.), ${ }^{6}$ ma la vicenda ci è nota grazie anche al

${ }^{6}$ Le edizioni più recenti del testo aramaico sono quelle di J. M. Lindenberger (ed.), The Aramaic Proverbs of Ahiqar (Baltimore, MD - London, 1983) pp. 41-275; I. KottsiePer, Die Sprache der Ahiqarsprüke (Berlin 1990); B. Porten - A. YArdeni (eds.), Textbook of Aramaic Documents from Ancient Egypt, newly copied, edited and translated into Hebrew and English (Jerusalem, 1993) vol. III: Literature, Accounts, Lists, pp. 22-57. Una traduzione italiana molto accurata e precisa, con spiegazione puntuale delle scelte critiche effettuate, si trova in R. ConTins, Il testo aramaico di Elefantina, in 
contributo delle altre traduzioni antiche, specialmente di quella siriaca, ${ }^{7}$ la più importante delle versioni complete, attestata in più recensioni. ${ }^{8}$

Questo scritto potrebbe forse essere considerato un romanzo. Tuttavia tale qualifica comporta una forzatura: infatti essa riconduce, entro le coordinate e i limiti di un genere letterario maturato nella tarda età ellenistica, a uno scritto molto più antico del romanzo greco, fortemente connotato dalla prospettiva didattico-sapienziale, che rimane comunque estranea a quest'ultimo. È tuttavia indubitato che la narrazione è condotta in forma romanzesca, in prima persona, con uno stile piano e immediato, che mette in rilievo la suspense e, nell'evocazione dello scenario del Vicino Oriente antico, lascia libero corso alla fantasia. Questo schema narrativo era particolarmente diffuso nel resto delle letterature sapienziali del mondo antico, ${ }^{9}$ dove possiamo trovare qualche precedente di quanto si legge in Ahiqar. ${ }^{10}$ Ma le analogie più interessanti, a livello di lessico

Contini - Grottanelli Il saggio Ahiqar pp. 113-139; essa si basa sull'edizione di Porten e Yardeni, ma si avvale anche dell'esame autoptico del manoscritto unico dell'Ahiqar di Elefantina, conservato nella Papyrussammlung di Berlino e catalogato come P. 13.446. Autorevole è anche la traduzione spagnola proposta da E. Martínez Borobio, «Libro arameo de Ajicar», in A. Dímz Macho et al. (eds.), Apócrifos del Antiguo Testamento. Con la colaboración de M. ${ }^{a}$ Angeles Navarro, A. de la Fuente, A. Piñero (Madrid, 2002; $2^{\mathrm{a}}$ ed.) vol. III, pp. 167-187: 177-187.

${ }^{7}$ Edita e tradotta da J. R. Harris, in F. C. Conybeare - J. R. HARris - A. S. Lewis (eds.), The Story of Ahikar, from the Aramaic, Syriac, Arabic, Armenian, Ethiopic, Old Turkish, Greek and Slavonic Versions (London - Cambridge - Glasgow, 1913; 2nd ed. enlarged and corrected) pp. 34-73; pp. 99-127.

${ }^{8}$ J. C. Vanderkam, Ahiqar (Book of), in D. N. Freedman et al. (eds.), Anchor Bible Dictionary (New York, 1992) vol. I, pp. 119a-120b: 119a.

${ }^{9}$ Greenfield «The Background and Parallel»pp. 50-51; Pennacchietti «Storia e massime» p. 53.

${ }^{10}$ Ad esempio, nella letteratura mesopotamica del viI sec. a.C. Cf. W. von SodEN, «Die Unterweltsvision eines assyrischen Kronprinzes. Nebst einigen Beobachtungen zur Vorgeschichte des Ahiqar-Romans», Zeitschrift für Assyriologie 43 (1936) N.F. pp. 1-31 (= W. von Soden, Aus Sprache, Geschichte und Religion Babyloniens: gesammelte Aufsätze [eds. L. CAGNI - H.-P. Müller; Napoli, 1989] pp. 39-67): 9-13. Così pure, le due tematiche di Ahiqar sono variamente elaborate e combinate fra loro nella storia del re sassonide Cosroe Anuširvan e del suo saggio ministro Buzurgmihr nel Libro dei Re di Firdousi, e nella leggenda indiana del re Nanda e del suo primo ministro Śakaala (A. J. KRAPPE, «Is the Story of Ahikar the Wise of Indian Origin?», Journal for the American Oriental Society 61 [1941] pp. 280-284). Cf. anche Pennacchietti «Storia e massime» p. 55. Secondo la tradizione greca anche Democrito di Abdera (460 a.C.) avrebbe tradotto Ahiqar. Fra il 30 e il 100 d.C. si data la Vita Aesopi, composta in Egitto sullo schema della vicenda di Ahiqar, a cui si 
e di pensiero, riguardano gli scritti biblici. Lo stesso Charles ${ }^{11}$ colloca Ahiqar fra gli apocrifi e pseudepigrafi della Bibbia ebraica. ${ }^{12}$ In Tobia il richiamo a questo libro è esplicito, infatti ne riprende il protagonista per farne, da saggio pagano, un pio osservante ebreo della tribù di Neftali, in esilio. ${ }^{13}$ Con ogni evidenza, con esiti diversi, le due tematiche dominanti di Ahiqar ispirarono l'agiografo nell'ideazione del suo racconto. Il primo tema è ripreso per narrare la vicenda di un pio osservante, colpito ingiustamente e poi riabilitato; il secondo è rielaborato in positivo nel racconto edificante della devozione di un figlio per il padre. Inoltre l'agiografo cita espressamente Ahiqar e ne rielabora la figura ai fini della vicenda che intende narrare. ${ }^{14}$

Quindi, accanto alle fonti dirette si debbono valutare attentamente anche le cosiddette fonti indirette, in cui la tradizione di Ahiqar è ripresa allusivamente o attraverso qualche isolata menzione. Per quanto limitato possa essere il loro apporto per la conoscenza di Ahiqar, esse forniscono un contributo certamente importante ai fini della ricostruzione della storia e dell'evoluzione della sua tradizione. Dopo il libro di Tobia, sono gli scritti in demotico a presentare le analogie più interessanti. Si tratta anzitutto di due frammenti di papiro del primo secolo d.C., entrambi riconducibili alla Storia di Ahiqar.

allacciano le avventure del saggio africano, schiavo presso la corte del re babilonese Licero (Conybeare - Harris - Lewis The Story of Ahikar pp. 162-167).

${ }^{11}$ R.H. Charles (ed.) The Apocrypha and Pseudepigrapha of the Old Testament in English, with Introductions and Critical and Explanatory Notes to the Several Books (Oxford, 1913; rist. 1964) vol. II: Pseudepigrapha, pp. 715-784.

${ }^{12}$ Cf. però le importanti precisazioni di Martínez Borobio «Libro arameo de Ajicar» p. 173, che conclude: «podemos catalogar a Ajicar como un texto perteneciente a la literatura de los pueblos del Próximo Oriente relacionada con los escritos del AT».

${ }^{13}$ Anche nel NT si trovano tracce del racconto di Ahiqar: in effetti è stato ipotizzato che la parabola del fico sterile (Lc 13,6.9) e quella del maggiordomo o del servo malvagio (Mt 24,45-51 // Lc 12,45-46) siano modellate su apologhi e massime di Ahiqar. Così la sorte di Giuda Iscariota in At 1,18-19 richiamerebbe quella di Nadin, e il proverbio di $2 \mathrm{Pt}$ 2, 22 riecheggerebbe uno degli apologhi di Ahiqar.

${ }^{14} \mathrm{Si}$ tratta notoriamente di un libro deuterocanonico o apocrifo, trasmesso principalmente in tre recensioni greche $\left(\mathrm{G}^{\mathrm{I}} \mathrm{-III}\right)$, complete le prime due, frammentaria la terza. L'edizione critica più recente è quella di R. Hanhart (ed.), Septuaginta. Vetus Testamentum Graecum Auctoritate Academiae Scientiarum Gottingensis editum (Göttingen, 1983) vol. VIII/5: Tobit. 
Dalla prima edizione critica (1976), ${ }^{15}$ attraverso ulteriori revisioni si è giunti a quella curata da Betrò, ${ }^{16}$ che segna un importante progresso nella pubblicazione del testo. Nei due frammenti di papiro sono rintracciabili vari elementi assenti dal testo elefantino e presenti, sia pure per accenni, nelle versioni successive. Si tratta del complotto di Nadin per far condannare Ahiqar per alto tradimento, e degli antecedenti del cosiddetto episodio egiziano, dove Esarhaddon espone ai suoi cortigiani le pretese esose del faraone. Tutto suggerisce quindi di datare questo testo a un'epoca intermedia fra la versione d'Elefantina e quelle successive. Sempre dall' ambiente egiziano proviene un secondo scritto, noto col nome di Istruzioni di Anchsheshonqi, ${ }^{17}$ il quale, secondo Lichteim, potrebbe riflettere Ahiqar e dipenderne. Tuttavia, poiché le modalità narrative delle due opere sono nettamente diverse, e perché la sezione gnomica di Anchsheshonqi affonda le sue radici prettamente nella tradizione egiziana, ancora con Betrò è preferibile credere che entrambe siano state (ri)elaborate in Egitto fra il sec. VII a.C. e l'età tolemaica in ambienti culturali che, anche se molto diversi, sono in un «rapporto di reciproca osmosi». Entrambi questi scritti in demotico sembrano riflettere una versione di Ahiqar intermedia tra il racconto elefantino e le rielaborazioni seriori. Questo potrebbe spiegare la presenza di numerosi elementi egiziani nelle versioni successive, e ben pochi invece nel testo ritrovato a Elefantina, che potrebbero risalire alla prima fase della sua importazione in Egitto.

${ }^{15}$ K.T. ZaUZICH, «Demotische Fragmente zum Achikar-Roman», in H. FranKE - W. HeIssig - W. Treue (eds.), Folia Rara Wolfgang Voigt LXV. Diem natalem celebranti... dedicata (Wiesbaden, 1976) pp. 180-185, con un contributo ulteriore in M. KüCHLER, Frühjüdische Weisheitstraditionen. Zum Fortgang weisheitlichen Denkens im Bereich des frühjüdische Jahweglaubens (Freiburg - Göttingen, 1979) pp. 333-337.

${ }^{16}$ M. Betrò, «La tradizione di Ahiqar in Egitto», in ContinI - GrotTANElli Il saggio Ahiqar pp. 177-191. Si tratta dell'edizione critica dei frammenti demotici relativi alla Storia di Ahiqar (pp. 188-191), a cui è premesso un saggio sulla tradizione di questo testo in Egitto (pp. 177-178).

${ }^{17}$ Edito da S. R. K. Glanville, Catalogue of Demotic Papyri in the British Museum (London, 1955) vol. II: The Instructions of Onchsheshonqy (British Museum Papyrus 10508) Part I: Introduction, Transliteration, Translation, Notes and Plates. Cf. anche H. J. Thissen, Die Lehre des Anchscheschonqi (P. BM 10508), Einleitung, Übersetzung, Indices (Bonn, 1984); K. RyHOLT, «A New Version of the Introduction to the Teachings of 'Onch-Sheshonqy», in P.J. Frandsen - K. Ryholt (eds.), A Miscellany of Demotic Texts and Studies, with contributions by J. F. QuACK (Copenhagen, 2000) pp. 113-140. 
Infine, ma a parte, andrà valutata anche un'iscrizione ritrovata a UrukWarka su una tavoletta babilonese datata al 165 a.C., ${ }^{18}$ che riporta un elenco di ummānu, cioè 'studiosi (di corte)', fra i quali è incluso anche Ahiqar, con un nome sumerico. Infatti, pur non riguardando strettamente la tradizione di Ahiqar, essa è stata a lungo ritenuta prova della storicità del saggio assiro.

Entro il IV sec. d.C. il libro di Ahiqar è tradotto dall'aramaico dell'impero in siriaco; dal $\mathrm{V}$ sec. le Massime compaiono in lingua armena, nella cui letteratura Ahiqar è giunto attraverso il siriaco. La versione siriaca (ms 2020 Cambridge, XVII sec.) mostra chiaramente l'appartenenza all'epoca pre-achemenide, assira o babilonese, tipica anche della versione aramaica di Elefantina. Infatti i nomi propri sono accadici, quelli dei funzionari appartengono all'aramaico di corte, cioè sono antichi, tanto da non esser più capiti nelle traduzioni successive. ${ }^{1}$

Il problema letterario si presenta in tutta la sua complessità già a una prima analisi. In effetti se è assodato che la narrazione più completa della vicenda di Ahiqar è quella trasmessa nella versione siriaca, è pur vero che il racconto è stato spesso rimaneggiato nel corso di due millenni con vari adattamenti. ${ }^{20}$

La critica ha raggiunto un accordo sostanziale sul luogo di composizione del libro, che pare la Mesopotamia, ma non è ancora chiaro se la lingua dell'originale fosse l'accadico o l'aramaico, e se già in epoca pre-achemenide (VII-VI sec. a.C.) esso avesse la medesima estensione. Il dubbio riguarda la sezione relativa all'incontro di Ahiqar con il faraone,

${ }^{18}$ Edita da G.-J. van DiJK, «Die Tontafeln aus dem reš-Heiligtum», in H. J. LenZeN (ed.), XVIII. vorläufiger Bericht über die Ausgrabungen in Uruk-Warka (= Vorläufige Berichte über die von dem Deutschen Archäologischen Institut und der Deutschen Orient-Gesellschaft aus Mitteln der Deutschen Forschungsgemeinschaft unternommen Ausgrabungen in Uruk-Warka) (Berlin, 1962) pp. 43-61: 45-51; tradotta da F. M. FALES, «Riflessioni sull'Ahiqar di Elefantina», Orientis Antiqui Miscellanea 1 (1994) pp. 39-60: $42 \mathrm{ss}$.

${ }^{19}$ Dalla siriaca dipendono due rami della tradizione testuale: 1) le recensioni armene A e B (V sec.), conservate in manoscritti dei secc. successivi al XV; la versione turca, scritta nel 1500 ca. (Crimea) e quella georgiana, derivate dall'armeno; 2) le versioni arabe, una delle quali è entrata nelle Mille e una notte; i frammenti etiopici; le versioni neo-aramaiche e quelle siriache, effettuate sull'arabo. A una versione greca risalgono varie recensioni slave (cf. Pennacchietti «Storia e massime» pp. 58-59).

${ }^{20}$ Pennacchietti «Storia e massime» pp. 54-55. 
che per vari studiosi parrebbe frutto di un'integrazione seriore. ${ }^{21}$ Del resto la versione più antica del libro, cioè quella d'Elefantina, non riporta l'episodio; tuttavia, con Pennacchietti, ${ }^{22}$ si può pensare che varie difficoltà di trasmissione testuale dell'aramaico siano all'origine dell'eliminazione dal testo di questa parte, come di altre.

In effetti, precisi rilievi linguistici sulla versione siriaca confermano che l'episodio egiziano apparteneva probabilmente all'originale. Pennacchietti menziona anzitutto la designazione della reggia del faraone con hayklä', «tempio», conforme all'originale aramaico e alla denominazione del palazzo di Esarhaddon nella versione di Elefantina e alla reggia di Nabucodonosor in Dn 4,1.26. Così pure, innanzi al faraone, Ahiqar paragona il suo re al dio $B^{\prime} e l$ Šamminn [sic], il «Signore del cielo», in grado di impedire al dio Bêl di «entrare e uscire in strada, e ai suoi dignitari di farsi vedere», tratti, questi, che richiamano la processione assira delle statue di Bêl durante le celebrazioni del Capodanno.

Il problema critico si complica ulteriormente nella sezione sapienziale del libro. In effetti la raccolta delle massime o proverbi della versione di Elefantina si distingue nettamente dalle successive. Essa è redatta in un aramaico che risente degli apporti di vari dialetti aramaici occidentali e orientali -diversamente dalla Storia, che attesta per lo più un aramaico imperiale, di carattere letterario-, cosa resa possibile dalla grande adattabilità del genere del proverbio e dalla sua diffusione in vari contesti popolari. ${ }^{23}$

Le due raccolte di massime, aramaica e siriaca, diverse per estensione (110 proverbi e apologhi, la prima; 75 proverbi più 41 detti, la seconda), dato che quella siriaca ha sviluppato successivamente altri proverbi, hanno in comune la dipendenza dalla stessa tradizione sapienziale di matrice aramaica, ma fortemente aperta all'influsso culturale assiro. Del resto anche la letteratura sapienziale ebraica -specialmente i Salmi e i

\footnotetext{
${ }^{21}$ L. PIrot, «Ahikar», in ID. (ed.), Supplément au Dictionnaire de la Bible (Paris, 1928) vol. I, pp. 198-207: 204.

${ }^{22}$ Pennacchietti «Storia e massime» p. 55.

${ }^{23}$ Cf. J. C. Greenfield, «The Dialects of Early Aramaic», Journal of Near Eastern Studies 37 (1978) pp. 93-99 (= PAul - Stone - Pinnick 'Al Kanfei Yonah pp. 160-166): 97; Lindenberger The Aramaic Proverbs pp. 19.279-304.
} 
Proverbi- si ricollega allo stesso filone siriaco e rivela varie affinità di stile e di concetti con Ahiqar.

Con Pennacchietti ${ }^{24}$ si può concludere che le Massime siano il frutto di un'aggiunta seriore alla Storia, fatta da scribi aramaici già nel VI-V sec. a.C.; questo processo contribuì enormemente alla loro diffusione, facendone un patrimonio comune a cui attinsero poi varie opere sapienziali successive, anche bibliche (per esempio Qohelet e Siracide). Le Massime sono consigli relativi alla vita sociale: esortano alla moderazione, alla prudenza, alla fedeltà e all'amicizia. Si tratta di una morale pratica, ispirata al culto dell'autorità che va riverita 0 , semmai, assecondata. ${ }^{25}$

Del resto, la stessa versione aramaica, restituita dai papiri lacunosi di Elefantina, dev'essere ritenuta già un adattamento dell'originale. ${ }^{26}$ Lo confermano vari aggiornamenti introdotti nel testo, come ad esempio il titolo ș]byt 'zqth, "guardasigilli», ${ }^{27}$ attribuito qui ${ }^{28}$ ad Ahiqar, che non può risalire oltre l'epoca achemenide: infatti nella traduzione siriaca gli corrisponde 'ezq $t \bar{a}$, «sigillo», ${ }^{29}$ abbreviazione dell' aramaico e, secondo Greenfield, ${ }^{30}$ calco dell'antico persiano o egiziano șbyt 'zqth, «latore del sigillo», detto di Ahiqar

\footnotetext{
${ }^{24}$ Pennacchietti «Storia e massime» p. 56.

${ }^{25}$ Esemplare, per questi aspetti, il proverbio $\mathrm{n}^{\circ} 12$, nello studio di A. Lemaire, «Le proverbe araméen Ahiqar 12», in J.-M. DuRAnd - J.-R. KuPPER (eds.), Miscellanea babyloniaca: Mélanges offerts à Maurice Birot (Paris, 1985) pp. 197-200.

${ }^{26}$ Pennacchietti «Storia e massime» p. 57.

${ }^{27}$ M. JAstrow, A Dictionary of the Targumim, the Talmud Babli and Yerushalmi, and the Midrashic Literature (London - New York, 1886-1903; $2^{\mathrm{a}}$ rist. anast. New York, 1950) vol. II, pp. 1886-1903: 1213a s.v. șebat; ibid. p. 1062a s.vv. 'izq $q^{e} t \bar{a}^{\prime}$ ' 'izqā' II; G. H. Dalman, Aramäisch-Neuhebräisches Handwörterbuch zu Targum, Talmud und Midrasch (Göttingen, 1938; $3^{a}$ ed., $2^{a}$ rist. anast. Hildesheim, 1997) p. 358b s.v. ș bat; ibid. p. 309b s.v. 'izqetā'.

${ }^{28}$ Porten - YARdeni Textbook of Aramaic Documents p. 26.

${ }^{29} \mathrm{~K}$. Brockelmann, Lexicon syriacum. Editio secunda aucta et emendata (Halis Saxonum, 1928; $2^{\mathrm{a}}$ rist. anast. Hildesheim 1995) p. 519b s.v. 'ezqe $t \bar{o}$ ', 'ezaqtō'.

${ }^{30}$ J. C. GREENFIELD, «Studies in Aramaic Lexicography I», Journal for the American Oriental Society 82 (1962) pp. 290a-299b: 292a-293b; 297a-299b. Cf. ibid. p. 293b: «It is therefore quite possible that the title byt ' $z q h$, as well as Ahiqar's other ones, are taken not from Assyrian court practices but from either Egyptian or Achamaenian usage. It is also probable that the version of Ahiqar circulating at Elephantine and read by the Jewish soldiers of the Persian garrison there had been modified or "modernized"».
} 
nel papiro di Elefantina. ${ }^{31}$ A questa versione aramaica, quindi, o ad un'altra perduta, si ispirò probabilmente l'autore di Tobia; Pennacchietti propone a conferma l'accezione specifica del vocabolo aramaico $s d q h$, «elemosina», a cui nel libro biblico si attribuisce carattere salvifico, mentre nella versione siriaca è reso con $k \bar{e} n \bar{u} t \bar{a}$, che significa semplicemente «rettitudine». ${ }^{32}$

\section{LA FORMAZIONE DELLA TRADIZIONE}

Recenti studi, basandosi sulle versioni antiche di Ahiqar, hanno riproposto il problema critico del libro e fornito ulteriori argomentazioni in vista dell'individuazione dell' originale. L'occasione è stata data da un contributo della classicista M. J. Luzzatto, che si è espressa in favore di un'origine accadica della Storia, in quanto il racconto più antico a noi noto, cioè la versione aramaica del papiro d'età achemenide rinvenuto a Elefantina, deriverebbe da un testo autobiografico in accadico, scritto «in cuneiforme, su stele» e databile al VII sec. a.C. ${ }^{33}$ Proprio le versioni siriache e armene attesterebbero questa origine assira; quella aramaica di Elefantina, invece, da cui dipendono tutte le successive versioni moderne, non rappresenterebbe una vera e propria «edizione», ma avrebbe carattere «antologico» e «scolastico», ${ }^{34}$ in quanto «testo documentario e celebrativo», ${ }^{35}$ concepito in «chiave di lettura moralistica, di impronta veterotestamentaria». ${ }^{36}$ Luzzatto perciò ritiene che, per risalire all' «originaria redazione assira», si dovrebbe procedere a un confronto con «il testo autobiografico celebrativo di imprese di re o di governatori mesopotamici [...], dall'arido elenco di campagne di guerra all'elaborata narrazione di eventi memorabili a scopo didattico», che «è legato alla

\footnotetext{
${ }^{31}$ Una rassegna dei vari titoli con cui è designato Ahiqar nell' aramaico di Elefantina si trova in J. C. Greenfield, «The Wisdom of Ahiqar», in J. DAY - R. P. Gordon - H. G. M. Williamson (eds.), Wisdom in Ancient Israel. Essays in Honour of J. A. Emerton (Cambridge, 1995) pp. 43-52: 44-45.

32 Pennacchietti «Storia e massime» p. 57. Quest'accezione è attribuita al termine siriaco nei proverbi ${ }^{\circ} 2$ e 37 della sezione seconda delle Massime.

${ }^{33}$ M. J. Luzzatto, «Grecia e Vicino Oriente: tracce della "Storia di Ahiqar" nella cultura greca tra vi e v secolo a.C.», Quaderni di Storia 36 (1992) pp. 5-84: 11.

${ }^{34} \mathrm{Cf}$. Luzzatto «Grecia e Vicino Oriente» p. 12.

${ }^{35}$ Luzzatto «Grecia e Vicino Oriente» p. 11.

${ }^{36}$ Luzzatto «Grecia e Vicino Oriente» p. 70 n. 33.
} 
stele (narû), spesso deposta in un tempio o a palazzo», destinata allo stesso ambiente di corte che l'ha prodotta. ${ }^{37}$

I limiti di quest'interpretazione sono stati segnalati anzitutto da F. M. Fales. Egli in particolare sottolinea che essa accosta indebitamente «generi testuali e storiografici completamente diversi del Vicino Oriente antico». ${ }^{38}$ In effetti nel primo millennio a.C., in ambiente mesopotamico, si hanno testi in accadico redatti in prima persona e scritti su stele, ma sempre a firma di personaggi pubblici dell'amministrazione dello Stato, per lo più di sovrani. Inoltre il genere letterario non è quello dell'autobiografia, ma piuttosto quello delle res gestae; quindi il racconto delle imprese militari e civili enumerate ha «scopo celebrativo e propagandistico». Del resto, a questa tipologia di narrazione risultano estranei sia il «resoconto di disgrazie personali» (o ingiustizie per mano di superiori), sia «l'appoggio di un apparato sapienziale o didascalico», volto «alla ricerca di un universo di valori etici». È vero che nella stessa epoca anche in ambito siriano e in aramaico si ha uno scritto di carattere storiografico e su stele, che narra in prima persona l'ascesa politica di un personaggio pubblico, la cui «passata disgrazia ha agito da premessa alla presente felicità e potenza». Ma, anche qui, chi narra è al vertice del potere politico, e «non ha bisogno di condire la storia della sua ascesa con proverbi o apologhi di saggezza». ${ }^{39}$ In definitiva Fales ritiene che l'autobiografia su stele, di carattere politico-sapienziale, supposta da Luzzatto, non esiste «né in accadico né in aramaico di epoca neo-assira»; quindi l'ipotesi comparativistica sostenuta per il romanzo di Ahiqar non trova documentazione nelle fonti vicino-orientali di questo libro. ${ }^{40}$ Questa critica è naturalmente condivisibile. ${ }^{41}$

${ }^{37}$ Luzzatto «Grecia e Vicino Oriente» p. 11.

${ }^{38}$ Luzzatto «Grecia e Vicino Oriente» p. 144.

${ }^{39}$ F.M. FALES, «Storia di Ahiqar tra Oriente e Grecia: la prospettiva dell'antico Oriente», Quaderni di Storia 38 (1993) pp. 143-166: 145.

${ }^{40} \mathrm{Si}$ vedano anche le puntuali osservazioni di R. ContinI, «I testi letterari aramaicoegiziani e l'Antico Testamento», in R. FABRIs (ed.), Il confronto tra le diverse culture nella Bibbia da Esdra a Paolo. XXXIV Settimana Biblica Nazionale (Roma, 9-13 settembre 1996), Ricerche Storico Bibliche 10 (1998) pp. 81-104: 83-84.

${ }^{41}$ Luzzatto è ritornata successivamente sulla questione con un ulteriore contributo (M. J. Luzzatto, «Ancora sulla "Storia di Ahiqar"», Quaderni di Storia 20 [1994] pp. 253-277) dove, pur aggiungendo interessanti puntualizzazioni, ribadisce la primitiva interpretazione, sulla quale si ripropongono perciò le riserve segnalate da Fales in rapporto al metodo adottato e ai risultati conseguiti. 
Con Fales ${ }^{42}$ si può quindi tentare una ricostruzione della tradizione testuale della Storia di Ahiqar in Assiria e nel mondo aramaico, «alla luce dei risultati più recenti». La prima edizione del testo risale al 1911, quando E. Sachau ${ }^{43}$ pubblicò alcuni papiri aramaici rinvenuti a Elefantina da una missione archeologica tedesca. Si tratta di un unico papiro, mutilo e frammentario, con resti di 14 colonne di testo. La riedizione critica del medesimo, ad opera di A. Cowley, ${ }^{4}$ è del 1923, e pone per la prima volta la questione dell'originale da cui dipende il papiro aramaico egizio del v sec. a.C. Infatti proprio i riferimenti ad Ahiqar in Tobia avevano fatto pensare a un'origine ebraica dell'opera; lo stesso suggeriva la sezione narrativa di quest'ultima, ambientata sotto Sennacherib e Esarhaddon, cioè nell' Assiria del VII sec. a.C., e così anche l'esistenza in Egitto della colonia militare achemenide, documentata nei papiri di Elefantina, la cui componente aramaica era affiancata da un elemento giudaico giunto prima del 550 a.C. ${ }^{45}$ Perciò, anziché studiare questo testo aramaico si erano cercate altre possibili fonti o antecedenti, preferibilmente un originale persiano, oppure accadico di età neo-assira.

Attualmente l'attenzione va piuttosto al ruolo dell'elemento aramaico nell'ambiente assiro dei secc. VIII-VII a.C. Le conoscenze acquisite negli ultimi due decenni hanno consentito di descrivere la fase tardiva dell'impero assiro come un'epoca caratterizzata da una «simbiosi assiro-aramaica», che vedeva la convivenza tra l'elemento linguistico assiro, «egemone ma statico» e ormai in crisi, e quello aramaico, subalterno ma in via di progressiva affermazione. ${ }^{46}$ In quest'orizzonte

${ }^{42}$ FALES «Storia di Ahiqar» pp. 145-149.

${ }^{43}$ E. Sachau, Aramäische Papyrus und Ostraka aus einer jüdischen Militärkolonie zu Elephantine. Altorientalische Sprachdenkmäler des 5. Jahrhunderts vor Chr. Generalverwaltung der Königlichen Museum zu Berlin (Leipzig, 1911) vols. I-II.

${ }^{44}$ A. E. Cowley, Aramaic Papyri of the Fifth Century B.C. (Oxford, 1923; rist. 1964) pp. 204-248.

${ }^{45}$ Per una valutazione sul piano storico-culturale dell'insediamento giudaico a Elefantina, rimane fondamentale l'opera di B. PORTEN, Archives from Elephantine: the Life of an Ancient Jewish Military Colony (Berkeley - Los Angeles, 1968). Ottimo strumento per lo studio della lingua di questi papiri è T. Muraoka - B. Porten, A Grammar of Egyptian Aramaic (Leiden, 1998), soprattutto dal punto di vista morfologico (ibid. pp. 42-153) e sintattico (ibid. pp. 155-211).

${ }^{46}$ FALES «Storia di Ahiqar» p. 147. Lo stesso utilizzo di un nuovo materiale scrittorio, cioè la pergamena o il papiro, più pratico per la scrittura alfabetica dell'aramaico, rispetto al cuneiforme delle tavolette, dovette agevolare questo «lento spostamento degli assi 
culturale si dovrebbe collocare la redazione dell'Ahiqar di Elefantina, che, del resto, contiene numerosi elementi linguistici propri del mondo aramaico d'Assiria (per esempio gli adattamenti dei nomi dei due re assiri, šn'ryb, per Sennacherib, e 'sr'dn, per Esarhaddon, o di quello di Nabû-šum-iškun, reso nbwsmskn), e calchi aramaici del lessico di corte assiro. ${ }^{47}$ Tutto ciò rinvia alle espressioni idiomatiche dell' ambiente di Ninive, documentate dalle lettere dei cortigiani del VII sec. a.C. ${ }^{48}$ Fales perciò conclude che il racconto della vicenda di un saggio dal nome aramaico Ahiqar, membro della corte di Sennacherib o Esarhaddon, sarebbe nato in Assiria nel VII sec. a.C.; dunque la Storia di Ahiqar sarebbe stata composta già in aramaico, probabilmente a Ninive, dove appunto questa lingua si andava sostituendo a quella assira nell'oralità e nella vita civile e giuridica, come testimoniano le lettere e i contratti. Due secoli dopo, questo racconto si sarebbe diffuso, sempre in aramaico, anche nell'Egitto achemenide, specialmente a Elefantina, grazie alla presenza in Egitto di Assiri, che erano venuti a contatto con quella colonia giudaica. In effetti i nomi propri assiro-aramaici che ricorrono nel papiro di Elefantina e anche l'epistolografia confermano che l'ambiente di Ninive e quello egizio erano legati sul piano giuridico, come ha dimostrato Y. Muffs. ${ }^{49}$ In tale contesto, nel v sec.

portanti linguistico-culturali dell'impero assiro» (ibid. p. 174). Sull'uso di questi materiali scrittori cf. H. TADMOR, «The Aramaization of Assyria: Aspects of Western Impact», in H. J. Nissen - J. Renger (eds.), Mesopotamien und seine Nachbarn: politische und kulturelle Wechselbeziehungen im Alten Vorderasien vom 4. bis 1. Jahrtausend v. Chr.: XXV. Rencontre Assyriologique Internationale, Berlin, 3. bis 7. Juli 1978 (Berlin, 1982) pp. 449-470: 451-453.

${ }^{47}$ F. M. FALES , «La tradizione assira ad Elefantina d'Egitto», Dialoghi di Archeologia 5 (1987) pp. 63-70: 68-69. Sull'onomastica dell'epoca neo-assira, cf. anche M. MARAQTEN, Die semitischen Personennamen in den alt- und reichsaramäischen Inschriften aus Vorderasien (Hildesheim, 1988); F. M. Fales, «West Semitic Names in the Assyrian Empire: Diffusion and Social Relevance», Studi Epigrafici e Linguistici sul Vicino Oriente antico 8 (1991) pp. 99-117.

${ }^{48}$ Cf. S. Parpola, The Royal Archives of Niniveh, in K. R. VeEnhof (ed.), Cuneiform Archives and Libraries: papers read at the $30^{\mathrm{e}}$ Rencontre Assyriologique Internationale Leiden, 4-8 July 1983 (Istanbul, 1986) pp. 223-226.

${ }^{49}$ Y. Murfs, Studies in the Aramaic Legal Papyri from Elephantine with Prolegomenon by Baruch A. Levine (Leiden, 2003). Cf. anche E. CussinI, The Aramaic Law of Sale and the Cuneiform Legal Tradition, Diss.: The Johns Hopkins University Press (Baltimore, MD, 1992) (tesi dottorale segnalata in FALES «Riflessioni sull' Ahiqar di Elefantina» p. 45 n. 41). 
a.C., ebbe origine la versione aramaica di Ahiqar, attestata dal papiro pubblicato da Sachau e da Cowley. ${ }^{50}$

\section{IL RIUSO DELLA TRADIZIONE}

Fra le prime attestazioni indirette di Ahiqar sono da annoverare i due frammenti di papiro in demotico con resti della sezione narrativa del libro, che risalgono al sec. I d.C. Il cattivo stato di conservazione rende molto difficile precisare a quale episodio della Storia si riferiscano esattamente, tuttavia è fuori discussione che essi forniscono un contributo di primaria importanza alla storia della tradizione, dato che costituiscono l'elemento di congiunzione tra il racconto elefantino e le versioni posteriori. Infatti, benché il testo restituito appaia frammentario, è possibile comprendere che si tratta di una versione della Storia che diverge sia dal testo di Elefantina che da quello delle rielaborazioni successive, alle quali tuttavia si mostra talora più vicino. ${ }^{51}$

Nel frammento del papiro del Cairo (A) si intravedono tracce dell'episodio delle trame ordite da Nadin contro il padre adottivo, fino al tranello teso ad Ahiqar. Questi due particolari, della calunnia e dell'inganno, assenti dal testo elefantino, saranno invece ripresi e ampliati poi nelle redazioni successive.

Nel frammento del papiro di Berlino (B) leggiamo invece il cosiddetto episodio egiziano, o meglio le sue premesse, cioè le richieste del faraone e l'incapacità dei dignitari assiri di esaudirle, seguite dal lamento sulla morte presunta di Ahiqar e dalla sua riabilitazione, una volta scoperta la finta morte. Abbiamo quindi una chiara attestazione che l'episodio egiziano doveva appartenere alla Storia di Ahiqar, o almeno alle sue redazioni della prima età romana, come precisa Betrò, incline a ritenere che lo si possa rintracciare -

${ }^{50}$ Un influsso così cospicuo e profondo del mondo assiro sulla cultura aramaicogiudaica d'Egitto, attestata da questa versione di Elefantina, si potrebbe spiegare, secondo FALES «Storia di Ahiqar» p. 149, soltanto «per via diretta», ipotizzando cioè che la colonia militare giudaica qui insediatasi avesse avuto origine «da un antico nucleo militare assiro in Egitto, stanziatosi al tempo dell'effimera conquista del Delta da parte dell'impero mesopotamico (ca. 668-663)».

${ }^{51}$ BETRÒ «La tradizione di Ahiqar» p. 177. 
grazie anche alla ricostruzione di Porten - Yardeni- probabilmente già nel testo aramaico d'Elefantina. ${ }^{52}$

Zauzich, che pubblicò nel 1976 il secondo dei due papiri, aggiunge che anche un terzo frammento papiraceo potrebbe offrire un apporto critico significativo per lo studio della tradizione ahiqariana. Si tratta di $P$. 15.658, proveniente forse dal Fayum, come il precedente, conservato a sua volta a Berlino e catalogato come $P .23 .729 .{ }^{53}$ Secondo Zauzich esso conterrebbe i resti di un testo sapienziale, nei quali si potrebbero intravedere alcune delle Massime di Ahiqar, in base alla grafia molto simile dei due papiri. ${ }^{54}$ Tuttavia, come precisa Betrò, ${ }^{55}$ nemmeno una frase vi è conservata per intero; inoltre uno solo è il passo di $P .15 .658$ che presenta affinità esplicite con le Massime, ma figura anche nelle Istruzioni di Anchsheshonqi. Quindi pare che il frammento appartenga a un testo sapienziale sconosciuto, probabilmente copiato dallo stesso scriba del P. 23.729 (il nostro frammento B). Del resto in Ahiqar la Storia e le Massime differiscono quanto ad origine e trasmissione. Ed è pure assodato che tra le Massime del testo elefantino e quelle delle versioni tarde esiste un rapporto piuttosto labile. L'ipotesi sostenuta attualmente dai critici è che le Massime furono aggiunte molto tardi alla Storia, in un fase piuttosto fluida del processo di trasmissione. Quindi i due frammenti papiracei (A e B) attesterebbero una versione della Storia che introduceva il testo sapienziale del $P .15 .658 .^{56}$

Un secondo testimonium indiretto in demotico è il già menzionato Anchsheshonqi. Questo scritto sapienziale presenta una struttura analoga ad Ahiqar, cioè una cornice narrativa che introduce una raccolta di massime. Lichtheim ha sostenuto la dipendenza di Anchsheshonqi da Ahiqar, ritenendo che l'autore conoscesse i detti del saggio assiro in

${ }^{52}$ Betrò «La tradizione di Ahiqar» p. 178. Porten - YARdeni Textbook of Aramaic Documents pp. 24-53; A. YARDENI, «Maritime Trade and Royal Accountancy in an Erased Customs Account from 475 B.c.E. on the Ahiqar Scroll from Elephantine», BASOR 293 (1994) pp. 67-78: 77 n. 9.

${ }^{53}$ ZAUZICH «Demotische Fragmente» pp. 180-185. Cf. anche Betrò «La tradizione di Ahiqar» p. 177 n. 1.

${ }^{54}$ ZAUZICH «Demotische Fragmente» p. 185 n. 20.

${ }^{55}$ Una prima decifrazione e traduzione del papiro si trova in Küchlen Frühjüdische Weisheitstraditionen pp. 333-337, poi ripresa anche da LINDENBERGER The Aramaic Proverbs pp. 310-312.

${ }^{56}$ BETRÒ «La tradizione di Ahiqar» p. 181. 
una versione molto più vicina a quella siriaca e a quelle tarde che non al racconto elefantino. ${ }^{57}$ Di fatto ha attinto ad Ahiqar come a un'antologia di massime sapienziali quasi senza apportarvi modifiche. Probabilmente poi conosceva anche la Storia, che faceva da cornice al libro di Ahiqar e inquadrava le Massime, e se ne servì per abbozzare la sua storia di Anchsheshonqi, e soprattutto il motivo del saggio prigioniero. ${ }^{58}$

Il papiro di Elefantina risale certamente all'Egitto del sec. v, quindi precede e fa da riferimento ad Anchsheshonqi. Inoltre Ahiqar era ben noto nel Vicino Oriente antico, dove l'aramaico era considerato la lingua internazionale. Per questo Lichtheim ritiene possibile postulare l'esistenza di una versione intermedia fra quella di Elefantina e quelle medievali, che precedette anche quelle d'età romana a cui risalgono $\mathrm{i}$ due frammenti in demotico. ${ }^{59}$ Perciò, se l'autore di Anchsheshonqi s'ispirò ad Ahiqar, lo lesse in una versione egiziana del sec. II a.C.

Ulteriore attestazione indiretta di Ahiqar è il libro biblico di Tobia. L'intertestualità delle due opere è assodata fin dal 1880, data che vide l'avvio di numerosi studi comparativi delle stesse. In effetti il saggio assiro è nominato espressamente in Tobia, ed è legato all'esilio di Tobi in Assiria, per la cui liberazione Ahiqar svolge un ruolo di primo piano, quale sovrintendente generale e responsabile della contabilità del regno (Tb 1,21). ${ }^{60}$

Il suo nome ricorre in sei menzioni-quattro esplicite e due criticamente più incerte- che scandiscono la storia narrata. ${ }^{61}$ In esse Ahiqar è presentato come un familiare di Tobi che intercede in suo favore presso il sovrano della corte assira per ottenergli il rientro in patria ( $\mathrm{Tb} 1,22)$, e lo aiuta

${ }^{57}$ M. Lichtheim, Late Egyptian Wisdom Literature in the International Context: a Study of Demotic Instructions (Freiburg - Göttingen, 1983) pp. 17-18.

${ }^{58}$ BETRÒ «La tradizione di Ahiqar» p. 182.

${ }^{59}$ Lichtheim Late Egyptian Wisdom Literature pp. 17-18.

${ }^{60}$ Sull'apporto della Storia di Ahiqar all'ideazione della vicenda di Tobi rimando al mio studio G. Toloni, «Tobi e Ahiqar. Sulle tracce di un racconto antico», in ConTINI Grottanelli Il saggio Ahiqar pp. 141-165: 159-165.

${ }^{61}$ Per un'esame dettagliato delle singole menzioni cf. i miei contributi: G. TolonI, «Ahiqar nel Libro di Tobia», Annali dell'Istituto Orientale di Napoli 71 (2011) pp. 87114; ID., «Ahiqar e Tobia. Intertestualità biblica di una tradizione assiro-aramaica», Annali dell'Istituto Orientale di Napoli 72 (2012) [in printing]. 
economicamente, data la confisca dei beni di Tobi decretata a seguito della sua fuga dalla città (Tb 2,10).

Il recente ritrovamento a Qumran di cinque manoscritti frammentari di Tobia, quattro in aramaico (4Q196-199) e uno in ebraico (4Q200), ${ }^{62}$ databili tra l'inizio del sec. I a.C. e la prima metà del I d.C., ${ }^{63}$ ha riproposto il problema critico del libro, imponendo quindi anche un riesame delle relazioni con Ahiqar. I manoscritti qumranici infatti testimoniano una redazione semitica, probabilmente aramaica, di Tobia, molto affine alla Vorlage della recensione greca lunga $\left(\mathrm{G}^{\mathrm{II}}\right)$, spesso confermata dalla Vetus Latina. ${ }^{64}$ Sebbene non si tratti dell'originale, è evidente che siamo in presenza di un testo scritto in un'età molto prossima alla redazione di Tobia. Infatti per la critica è acquisito che il libro dovrebbe datarsi in un'epoca compresa tra il 220 e il 175 a.C. (forse verso la fine di questo periodo). ${ }^{65}$ Anche l'agiografo di Tobia, che probabilmente compose la sua opera in aramaico, dovette conoscere il testo aramaico di Ahiqar, data l'ampia notorietà di quest'ultimo nel Vicino Oriente antico, quindi alla Storia si ispirò almeno nell'ideazione del suo racconto.

Le citazioni esplicite $(1,21-22 ; 2,10 ; 11,19 ; 14,10.15)$ di fatto presuppongono nel lettore la conoscenza della Storia di Ahiqar, a cui alludono con precisi accenni evocativi. L'immagine rielaborata di Ahiqar

${ }^{62}$ Editi inizialmente in K. BEYER (ed.), Die aramäischen Texte vom Toten Meer. Ergänzungsband (Göttingen, 1994) pp. 134-147, in cui l'ordine degli avvenimenti narrati è conforme al $\mathrm{G}^{\mathrm{II}}$, i frammenti qumranici apparvero poi nell'editio princeps di J. A. FitzMYer (ed.), «Tobit», in M. Broshi et al. (eds.), Qumran Cave 4 (Oxford, 1995) vol. XIV/2: Parabiblical Texts, pp. 1-76, pls. I-X, dove invece si distinguono i manoscritti aramaici da quello ebraico. Molto affine ad essa è l'edizione di F. GARCía MartíneZ - E. J. C. TigchelaAR (eds.), The Dead Sea Scrolls Study Edition (Leiden - New York - Köln, 1997) vol. I: $1 Q 1-4 Q 273$, pp. 382-299.

${ }^{63}$ J. A. Fitzmyer, The Aramaic and Hebrew Fragments of Tobit from Qumran Cave 4», Catholic Biblical Quarterly 57 (1995) pp. 655-675 (= ID., The Dead Sea Scrolls and Christian Origins [Grand Rapids, MI, 2000] pp. 131-158): 657.

${ }^{64} \mathrm{Sul}$ confronto critico dei vari rami della tradizione, in vista della restituzione dell'originale, rimando alla mia monografia G. Toloni, L'originale del libro di Tobia. Studio filologico-linguistico (Madrid, 2004). Sull'importanza dei frammenti per la critica testuale di Tobia cf. J. A. FItZMYER, «The Significance of the Hebrew and Aramaic Texts of Tobit from Qumran for the Study of Tobit», in L. H. Schiffman - E. Tov - J. C. Vanderkam (eds.), The Dead Sea Scrolls: Fifty Years after their Discovery. Proceedings of the Jerusalem Congress - July 20-25 1997 (Jerusalem, 2000) pp. 418-425.

${ }^{65}$ J. A. FitzMYer, Tobit: Introduction, Commentary, and Notes (Berlin - New York, 2003) p. 52. 
è quella di un parente di Tobi (in 1,21-22 un nipote; in 11,19 un cugino), in alto rango alla corte di Esarhaddon. In 1,21-22 la rievocazione dei titoli ufficiali di Ahiqar nei frammenti qumranici riprende solo in parte quelli dei funzionari della corte assira citati nella Storia di Ahiqar; invece quelli che appaiono nelle recensioni greche riflettono piuttosto il linguaggio amministrativo tolemaico. Nell'ultima menzione, poi, si notano due novità rispetto alla Storia: anzitutto, come nelle versioni tarde, si indica esplicitamente il nascondiglio sotterraneo di Ahiqar, presunto morto, inoltre si attribuisce a Ahiqar la pratica dell'elemosina. Questo funge da conferma ulteriore del noto presupposto di Greenfield, cioè che la versione di Ahiqar utilizzata dall'agiografo di Tobia era già stata rielaborata rispetto alla formulazione originale, e si avvicina invece alle versioni tarde, in particolare alla siriaca antica, designata come «siriaca A». ${ }^{66}$

Perciò Tobia è presentato come l'antitesi di Nadin: mentre quest'ultimo tradisce il padre adottivo, egli diventa esempio di amor filiale. Anche l'aspirazione ad avere una pia sepoltura, motivo di Tobia, è presente nella Storia; invece l'elemosina attribuita ad Ahiqar nel libro biblico potrebbe dipendere dal fraintendimento del sostantivo șidq $\bar{a}^{\prime} / s^{e} d a q t \bar{a}^{\prime}$ che significa anche «rettitudine», prerogativa di Ahiqar in Massime 8,37.

Il riuso della tradizione ahiqariana, evidente grazie ai suoi riflessi letterari, è strettamente connesso con la storia della sua trasmissione. In effetti la trama della leggenda che affiora nelle primitive attestazioni, successive alla versione aramaica elefantina, mostra una notevole variazione sia nella struttura del racconto, che negli elementi narrativi utilizzati. Questa serie di modifiche rispetto alla forma più antica induce Lindenberger ${ }^{67}$ a proporre un'ipotesi di «stemma» (poi precisata da Fales ${ }^{68}$ in rapporto agli stadi pre-cristiani dello sviluppo della tradizione) in cui, fra la versione aramaica d'Elefantina e i primi riusi significativi, come i frammenti in demotico e Tobia, si deve presupporre

${ }^{66}$ J. C. Greenfield, «Two Proverbs of Ahiqar», in T. Abusch - J. Huehnergard - P. SteINKeller (eds.), Lingering over Words: Studies in Ancient Near Eastern Literature in Honor of William L. Moran (Atlanta, GA, 1990) pp. 195-201 (= PAUL - Stone - PinNicK 'Al Kanfei Yonah pp. 313-319): 199. La versione siriaca è pervenuta in cinque recensioni (A-E); con la lettera $\mathrm{A}$ si indica quella comunemente ritenuta «la più antica e attendibile» (R. Contini, «Introduzione», in Contini - Grottanelli Il saggio Ahiqar pp. 11-89: 36). Una sua accurata traduzione, con annotazioni puntuali, è data in PenNacchietTI «Storia $\mathrm{e}$ massime» pp. 65-95.

${ }^{67}$ Lindenberger The Aramaic Proverbs pp. 4-7.

${ }^{68}$ FALES «Riflessioni sull' Ahiqar di Elefantina» pp. 51-58. 
una versione aramaica «elaborata», da cui si sarebbero diramati un filone occidentale e uno orientale, entrambi collocabili in una fase che precede almeno di due secoli l'era cristiana. Dal primo proverrebbe una versione greca «trasfomata» nella sezione babilonese della Vita Aesopi, ${ }^{69}$ e starebbe all'origine delle traduzioni dell'Europa orientale; dal secondo, invece, verrebbe una versione siriaca «originaria», a cui andrebbero ricondotte le versioni asiatiche, e anche i riflessi della tradizione in Tobia e nei frammenti in demotico. Questa versione aramaica «elaborata» doveva costituire un testo ancora molto fluido e aperto a rielaborazioni; ad essa anzitutto, ma anche alle due versioni greca e siriaca «originaria», debbono esse attribuite le innovazioni delle versioni tarde rispetto al racconto elefantino.

Quindi i frammenti in demotico e Tobia confermano inequivocabilmente l'evoluzione della tradizione di Ahiqar, che ha intensificato la presenza di elementi novellistici e romanzeschi, tollerando nella trama qualche incongruenza, ridondanza e integrazione, talora non necessaria alla comprensione del racconto. Quanto ad Anchsheshonqi, non è attestata una dipendenza da Ahiqar. I due scritti vanno esaminati invece alla luce dell'internazionalità della cultura dell'Egitto tardo, nella cui società cosmopolita scambi e prestiti culturali erano frequenti. Ciò spiega le varie analogie di Anchsheshonqi con Ahiqar. Del resto in questo scritto in demotico alcuni elementi essenziali di Ahiqar sono assenti o utilizzati diversamente: in particolare il motivo della riabilitazione dopo la disgrazia, quello della morte apparente o della salvezza ottenuta in extremis quale ricompensa della rettitudine passata, quello dell'ingratitudine e del tradimento. Qui è avvenuto uno slittamento di alcuni elementi narrativi fondamentali, spostati dalla figura del protagonista al rapporto tra il medico Horsiesi e il faraone, con uno «sdoppiamento della figura centrale del racconto», ${ }^{70}$ difficilmente comprensibile se il modello da imitare era Ahiqar. Anchsheshonqi si data fra il sec. VII e l'età tolemaica, perciò non è più recente di Ahiqar; anzi, potrebbe anche aver contribuito alla nascita della versione egiziana di Ahiqar, dalla quale dipendono le versioni recenti. Quindi, più che di una dipendenza di Anchsheshonshqi da Ahiqar, dovette trattarsi dello sviluppo parallelo delle due opere, avvenuto in ambienti culturali comunicanti ma distinti.

Particolare importanza assume quindi l'episodio egiziano di Ahiqar, che sembra un racconto antico ma rielaborato con fini e mezzi diversi,

${ }^{69}$ Contini «Introduzione» p. 38.

70 BETRÒ «La tradizione di Ahiqar» p. 183. 
sostituendo alla magia la potenza dell'abilità retorica e dell'arguzia. La ripresa e la rielaborazione in Ahiqar dei vari temi egiziani avvenne perciò in un ambito culturale ben integrato in Egitto, pur avendo avuto un'origine diversa, cioè la comunità egiziana di lingua aramaica. A Elefantina infatti ebbe luogo un'osmosi aramaico-egiziana riscontrabile nelle istituzioni giuridiche, nelle relazioni sociali e nell'onomastica. ${ }^{72}$ Lo attesta chiaramente il $P$. Amherst 63, un testo letterario e religioso aramaico ma scritto in demotico. ${ }^{73}$

Quindi, probabilmente la Storia originaria di Ahiqar, dopo il sec. V (epoca in cui fu copiato l'Ahiqar di Elefantina) si arricchì di elementi tra la conquista assira dell'Egitto e la prima età romana, a cui risalgono i frammenti in demotico.

\section{DAlla LEGGENDA ALlA STORIA}

Il diverso impianto ideologico del testo elefantino rispetto alle versioni tarde induce -con Contini- a individuare il suo genere letterario nella cosiddetta leggenda di corte, diffusa in tutto il Vicino Oriente antico e in particolare nell'impero achemenide, come attesta lo stesso Anchsheshonqi. Questo genere letterario internazionale è presente anche nella letteratura giudaica, poiché appartiene propriamente a una serie di scritti dei secc. V-IV a.C. poi rielaborati in libri biblici d'età ellenistica. Si pensi, per esempio, al libro di Daniele (cc. I-VI), Susanna e Bel e il dragone, al Proto-Ester, e alla Storia

${ }^{71}$ Il rapporto tra retorica e magia ha attirato da tempo l'attenzione degli studiosi. In particolare ha caratterizzato la linea di ricerca di R. RITNER, a partire dalla sua celebre monografia The Mechanics of Ancient Egyptian Magical Practice (Chicago, 1993). Ma cf. anche ID., «Egyptian Magic: Questions of Legitimacy, Religious Orthodoxy and Social Deviance», in A. B. Lloyd (ed.), Studies in Pharaonic Religion and Society in Honour of J. Gwyn Griffiths (London, 1992), pp. 189-200; e ID., «Religion vs. Magic. The Evidence of the Magical Statue Bases», in U. LuFt (ed.), The Intellectual Heritage of Egypt. Studies Presented to Lázló Kákosy by Friends and Colleagues on the Occasion of his $60^{\text {th }}$ Birthday (Budapest, 1992) pp. 495-501.

72 Sulla letteratura aramaica ritrovata in Egitto nella biblioteca giudaica di Elefantina rinvio al contributo di R. ConTINI, «I documenti aramaici dall'Egitto persiano e tolemaico», Rivista Biblica 34 (1986) pp. 73-109.

${ }^{73}$ R. C. STEINER, «Papyrus Amherst 63: A New Source for the Language, Literature, Religion, and History of the Aramaeans», in M. J. Geller et al. (eds.), Studia Aramaica: New Sources and New Approaches. Papers Delivered at the London Conference of the Institute of Jewish Studies University College - London, $26^{\text {th }}-28^{\text {th }}$ June 1991 (New York, 1995) pp. 199-207. Cf. anche ConTINI «I testi letterari» p. 81. 
di Giuseppe (Gn 37.39-50), che, con le versioni tarde di Ahiqar, attestano specificamente il genere della novella o proto-romanzo. ${ }^{74}$ Propriamente, in Tobia il genere sta a metà fra la novella e il romanzo didattico-sapienziale, pur con una netta preponderanza degli elementi del secondo. Tutte queste leggende sono però accomunate dal ruolo del loro protagonista, che è quello del rappresentante positivo di un gruppo etnico integrato in un grande impero governato da un sovrano straniero. Da qui partono le varie analogie della struttura narrativa di questi scritti con la vicenda di Ahiqar.

Ma la stessa leggenda del saggio assiro potrebbe aver rielaborato, a sua volta, un antico racconto affine. Fales fa notare che la ricerca prosopografica ha rivelato l'esistenza in Assiria di un possibile antecedente di Ahiqar: si tratta della storia di Adad-šumu-uur -anziano esorcista di Esarhaddon-e del figlio Urad-Gula. Dopo la morte di Esarhaddon (668), con l'insediamento di Assurbanipal si ha un rinnovo delle cariche palatine; ma Urad-Gula resta escluso per ragioni politiche dalla successione al padre nel ruolo di esorcista. Adad-šumu-uur, «uno dei più abili e stimati specialisti» della corte reale e «discendente di una famiglia di scribi e studiosi», ${ }^{75}$ tenta perciò con una lettera di riproporre la candidatura del figlio al nuovo sovrano, il quale garantisce che accoglierà la sua richiesta. Tuttavia, molti anni dopo, Urad-Gula, ormai anziano a sua volta, in una lettera ad Assurbanipal rende noto di non aver avuto la nomina promessa, dato che si ritrova «povero in canna e maltrattato da tutti [...] senza l'adeguato compenso dal re». ${ }^{76}$ L'autore di Ahiqar potrebbe aver ricalcato questo antecedente, e così scrivere la sua Storia. È vero infatti che il menzionato epistolario offre interessanti analogie sia sul piano strutturale che su quello degli elementi espressivi; ${ }^{77}$ ma la vicenda di Ahiqar e Nadin, ci assicura che, pur non potendo escludere questa possibile interferenza di tematiche e motivi, non vi sono elementi a sostegno di una dipendenza letteraria, trattandosi di due opere nettamente diverse. Per Fales, dunque, la Storia di Ahiqar è una rielaborazione letteraria, che trasformò la famosa leggenda dell'esorcista assiro in un romanzo sui grandi temi di quell'epoca: giustizia e retribuzione, potere reale e autorevolezza del sapiente, condizione dell'anziano e ruolo del giovane. ${ }^{78}$

\footnotetext{
${ }^{74}$ CONTINI «I testi letterari» pp. 88-89, 102.

${ }^{75}$ FALES «Storia di Ahiqar» p. 150.

${ }^{76}$ FALES «Storia di Ahiqar» p. 151.

${ }^{77}$ Analizzati efficacemente in Fales «Storia di Ahiqar» pp. 151-152.

${ }^{78}$ FALES «Storia di Ahiqar» p. 152.
} 
In Tobia perciò avvenne un processo analogo, che comportò la «storicizzazione» di Ahiqar. Infatti la figura del saggio assiro è entrata di fatto esplicitamente nel racconto dell'agiografo, esigendo vari adattamenti e trasposizioni, col risultato di dare una prospettiva «storica» alla leggenda di Ahiqar. Come si è accennato sopra, questo processo è attestato anzitutto dalla rilettura della titolazione di Ahiqar adottata nelle diverse recensioni del libro: più vicina a quella tolemaica ${ }^{79}$ nel $G^{\mathrm{II}}$ e nella Vetus Latina, e più semplificata rispetto alla Storia di Ahiqar in 4Q199-200. Il tentativo soggiacente a questi adattamenti lessicali è quello di «storicizzare» la figura del saggio assiro, caratterizzata da tratti diversi nelle varie tradizioni ahiqariane.

Il rapporto fra Ahiqar e Tobia non è però quello di una stretta dipendenza letteraria, ${ }^{80}$ dato che l'autore biblico non abbozzò la vicenda di Tobi sul modello di quella di Ahiqar, bensì sfruttò la conoscenza di questo celebre racconto popolare presso i suoi lettori per dare prestigio al suo libro. ${ }^{81}$ Ahiqar godeva infatti di una notorietà internazionale, essendo l'aramaico la lingua ufficiale delle relazioni in tutto il Vicino Oriente antico. Pertanto anche le comunità giudaiche della diaspora egiziana -in particolare quella di Elefantina nel sec. V a.C.- conoscevano certamente la vicenda del saggio assiro. Un autore giudaico, in epoca ellenistica, adattò quest'opera, conosciuta in una forma già più elaborata rispetto alla versione elefantina, alla tradizione giudaica, facendone un romanzo sapienziale. ${ }^{22}$ Quindi, egli si avvalse della fama della Storia di Ahiqar, «storicizzando» la figura del protagonista, e inserendolo tra i personaggi di Tobia.

${ }^{79}$ CONTINI «I testi letterari» p. 92.

${ }^{80}$ Così invece J. C. DAncy, «Tobit», in J. C. DAnCy - W. J. Fuerst - R. J. Hammer (eds.), The Shorter Books of Apocrypha: Tobit, Judith, Rest of Esther, Baruch, Letter of Jeremiah, Additions to Daniel and Prayer of Manasseh, in P. R. ACKROYD - A. R. C. Lerner - J. W. PARKer (eds.), Cambridge Bible Commentary on the New English Bible (Cambridge, U.K., 1972) pp. 1-66: 6.

${ }^{81}$ È la nota tesi di J. C. Greenfield, «Ahiqar in the Book of Tobit», in J. Doré - P. Grelot - M. CARrez (eds.), De la Tôrah au Messie. Études d'exégèse et d'herméneutique bibliques offertes à Henri Cazelles (Paris, 1981) pp. 329-336 (= PAUL - STONE - Pinnick 'Al Kanfei Yonah pp. 195-202).

${ }^{82}$ Sui rapporti e i reciproci influssi tra i due ambiti letterari, giudaico e aramaico, rimando a A. LEMAIRE, «Aramaic Literature and Hebrew Literature: Contacts and Influences in the First Millennium B.C.E.», in $9^{\text {th }}$ Word Congress of the Jewish Studies, Panel Session: Hebrew and Aramaic Languages (Jerusalem, 1988) pp. 9-24. 
Un'ulteriore interessante menzione di Ahiqar si ha in una lista ritrovata ad Uruk-Warka negli scavi dell'inverno del $1959-1960^{83}$ e datata 16.5.165 a.C., ${ }^{84}$ dove si citano saggi (apkallū) e dotti (ummānū). Tra questi ultimi figura un $a$-ba-d.nin-nu-da-ri, nome sumerico ma da leggersi in accadico come corrispondente a Mannu-kima-Enlil-hatin, un ummānu d'età medio babilonese, il quale rimanda a un intellettuale vissuto a Babilonia 500 anni prima di Esarhaddon. Nella lista se ne dà il corrispettivo aramaico: Ahu'aqari, cioè Ahiqar. Si tratta, perciò, di una «identificazione apocrifa»: ${ }^{85}$ con essa i dotti di Uruk-Warka pongono l'ummānu Ahiqar sotto Esarhaddon e in Assiria. ${ }^{86}$ Questo procedimento ha lo stesso valore dell'operazione compiuta dall'agiografo di Tobia: è «promozione "per chiara fama"» di Ahiqar, che in Tobia e nella lista di Uruk-Warka diventava «protagonista della Storia», non più solo «di una storia». ${ }^{87}$

È quindi evidente che quest' iscrizione non può certo più esser addotta per provare l'esistenza storica di Ahiqar, ${ }^{88}$ e tanto meno la redazione in accadico della Storia di cui questi è protagonista, dato che il documento costituisce un caso di «storicizzazione spuria», ${ }^{89}$ molto simile a quella attuata dall'agiografo di Tobia.

Nel contempo ciò conferma che la vicenda di Ahiqar era ormai di dominio internazionale, e che il papiro di Elefantina dava una versione che collimava con quella diffusa, perché ambientava la vicenda sotto Esarhaddon, come avviene nella stessa lista di Uruk-Warka, non sotto Sennacherib, come si avrà nelle versioni successive.

Recibido: 01/12/2012

Aceptado: 28/05/2013

${ }^{83}$ GReEnFIELD «The Wisdom of Ahiqar» p. 44.

${ }^{84}$ Pubblicata da VAN DiJK «Die Tontafeln» pp. 44-52.

${ }^{85}$ FALES «Storia di Ahiqar» p. 155.

${ }^{86}$ Sul tema si veda lo studio di H. CAZELLES, «Ahiqar, ummân and amun and biblical wisdom texts (Pr. 8,30)», in Z. Zevit - S. Gitin - M. SoKoloff (eds.), Solving Riddles und Untying Knots. Biblical, Epigraphic, and Semitic Studies in Honor of Jonas C. Greenfield (Winona Lake, IN, 1995) pp. 45-55 (= H. CAzELLES, «Ahiqar, ummân and amun», in ID., Études d'histoire religieuse et de philologie biblique [Paris, 1996] pp. 243-252).

${ }^{87}$ FALES «Storia di Ahiqar» p. 155.

${ }^{88}$ PARPOLA, «Il retroterra assiro di Ahiqar», in CONTINI - GrotTANelli Il saggio Ahiqar pp. 91-112.

${ }^{89}$ Cf. anche CONTINI «Introduzione» p. 14. 